\title{
Chaihu-Shugan-San Decoction Modulates Intestinal Microbe Dysbiosis and Alleviates Chronic Metabolic Inflammation in NAFLD Rats via the NLRP3 Inflammasome Pathway
}

\author{
Yinji Liang $\mathbb{D},{ }^{1,2}$ Yupei Zhang, ${ }^{2}$ Yuanjun Deng, ${ }^{2}$ Shu Liang, ${ }^{2}$ Yifang He, ${ }^{2}$ Yanning Chen, \\ Chan Liu, ${ }^{3}$ Chenli Lin, ${ }^{3}$ Li Han, ${ }^{4}$ Guifang Tu, ${ }^{2}$ and Qinhe Yang $\mathbb{D}^{2}$ \\ ${ }^{1}$ School of Nursing, Jinan University, No. 601 Huangpu Avenue West, Guangzhou, Guangdong 510632, China \\ ${ }^{2}$ School of Traditional Chinese Medicine, Jinan University, No. 601 Huangpu Avenue West, Guangzhou, Guangdong 510632, China \\ ${ }^{3}$ Department of Pathology, School of Basic Medicine, Jinan University, No. 601 Huangpu Avenue West, Guangzhou, \\ Guangdong 510632, China \\ ${ }^{4}$ Department of Traditional Chinese Medicine, First Affiliated Hospital, Jinan University, No. 613 Huangpu Avenue West, \\ Guangzhou, Guangdong 510632, China
}

Correspondence should be addressed to Qinhe Yang; tyangqh@jnu.edu.cn

Received 21 October 2017; Revised 8 February 2018; Accepted 27 June 2018; Published 11 July 2018

Academic Editor: Omer Kucuk

Copyright (C) 2018 Yinji Liang et al. This is an open access article distributed under the Creative Commons Attribution License, which permits unrestricted use, distribution, and reproduction in any medium, provided the original work is properly cited.

\begin{abstract}
We evaluate the effects of the Chaihu-Shugan-San decoction on intestinal microbe dysbiosis and chronic metabolic inflammation via the NLRP3 pathway in NAFLD rats that were fed a high-fat diet. Twenty-four SD rats (male, six weeks old, $200 \pm 20$ g) were randomly divided into three groups: normal control group (NC group), high-fat diet-fed group (HFD group), and Chaihu-ShuganSan decoction intervention group ( $\mathrm{CH}$ group). The NC group rats were given standard feed, the HFD group rats were all fed a highfat diet $(83 \%$ standard feed $+10 \%$ lard oil $+5 \%$ sucrose $+1.5 \%$ cholesterol $+0.5 \%$ cholate), and the $\mathrm{CH}$ group rats were given a HFD plus Chaihu-Shugan-San at $9.6 \mathrm{~g} \cdot \mathrm{kg}^{-1} \bullet \mathrm{d}^{-1}$. Body composition, serum and liver lipids, inflammatory markers, intestinal microbial population, and the NLRP3 pathway-associated protein were assessed. The results showed that Chaihu-Shugan-San decoction significantly reduced body weight and total fat mass and the levels of serum LPS, TG, TNF- $\alpha$, IL- $1 \beta$, and IL-18, as well as liver TC, TG, TNF- $\alpha$, IL- $1 \beta$, and IL-18 $(P<0.05)$. The abundance of Enterobacteriaceae $(0.375 \%$ versus $0.064 \%, P<0.05)$, Staphylococcaceae families $(0.049 \%$ versus $0.016 \%, P<0.05)$ and Veillonella genus $(0.096 \%$ versus $0.009 \%, P<0.01)$ significantly decreased, whereas the abundance of Anaeroplasma genus $(0.0005 \%$ versus $0.0178 \%, P<0.01)$ significantly increased. The expression levels of NLRP3, ASC, and Caspase- 1 were changed significantly $(P<0.05)$. In summary, the Chaihu-Shugan-San decoction modulated intestinal microbe dysbiosis, reduced fat accumulation, and alleviated inflammatory factor expression, which are all processes related to the NLRP3 inflammasome pathway in NAFLD rats.
\end{abstract}

\section{Introduction}

Modern medical studies have suggested that nonalcoholic fatty liver disease (NAFLD) is a manifestation of metabolic syndrome (MS) in the liver that is often associated with obesity, dyslipidemia, and insulin resistance (IR)[1]. In recent years, the incidence and prevalence of NAFLD have increased, and it is one of the most common chronic liver diseases worldwide [2]. In Europe and the United States, the incidence rate of adult NAFLD was $20 \%-30 \%$ [3], whereas the incidence was $15 \%-30 \%$ in China [4]. NAFLD is indicated by more than $5 \%$ liver fat accumulation in imaging and/or histological pathology, and $25 \%-59.10 \%$ of NAFLD patients progress to nonalcoholic steatohepatitis (NASH) $[5,6]$. NASH is the most serious pathological condition associated with NAFLD, and NASH leads to hepatocyte inflammatory injury, which can develop into cirrhosis or hepatocellular carcinoma (HCC). Despite being viral infection, large amounts of alcohol intake over a long-term period and other causes explain how the majority of cirrhosis cases arise; a small portion of cases still lack a clear cause and are categorized as cryptogenic cirrhosis (CC) [7]. Some studies 
have found that approximately $50 \%$ of CC cases arise from NASH [8], and even a significant proportion of CC patients (15-30\%) eventually progress to liver failure and HCC $[9,10]$. Furthermore, NAFLD causes or aggravates cardiovascular and cerebrovascular diseases, diabetes, cancer, and other diseases [5].

Inflammation is one of the most important factors in the pathogenesis of NAFLD, and activation of the NLRP3 inflammasome was considered to be the key link to hepatocyte injury, immune cell activation, and expansion of liver inflammation in NAFLD [11]. NLRP3 is currently thought to be activated by various types of pathogen-associated molecular patterns (PAMPs) or damage-associated molecular patterns (DAMPs), especially in hepatocytes and immune cells. Activation of the NLRP3 inflammasome required two links [12]. First, activation of the LPS/TLR4 pathway is the first signal necessary to activate NF- $\kappa \mathrm{B}$, which increases the expression of NLRP3 and IL- $1 \beta$ precursors. Second, activation of Caspase- 1 and the release of IL- $1 \beta$ and IL-18 eventually lead to a "waterfall" inflammatory cascade and cause cell damage. Recent studies have demonstrated that the intestinal microenvironment also plays an important role in NLRP3-mediated liver injury in NAFLD. However, the specific mechanism has not yet been elucidated. Many studies have confirmed that there is a significant difference in the composition and function of the intestinal microbiota between NALFD patients and healthy individuals. Shanab AA et al. [13] found that the prevalence of enteric bacterial overgrowth syndrome (EBOS) in NASH patients was significantly higher than that in healthy controls. It has also been found that the proportion of the Ruminococcaceae family of the intestinal microbiota in the fecal samples of NAFLD and NASH patients was lower than that in healthy subjects [14]. In addition, the proportion of Clostridium coccoides in adult NASH patients was significantly higher than that in NAFLD patients [15]. It has also been found that the severity of NAFLD was significantly and negatively correlated with the abundance of $\gamma$-Proteus in the intestine [16]. Although some of the data are controversial, these studies indicated that certain bacteria may be beneficial or harmful to patients with NALFD.

Currently, the prevention and treatment of NAFLD are common in western medicine and traditional Chinese medicine (TCM). New guidelines suggest that health education and lifestyle interventions are still the preferred treatment for NAFLD/NASH. In light of this treatment approach, single herbal medicines, compound TCM and its active ingredients for the prevention, and treatment of NAFLD have become popular topics in this field. It has been found that some TCM compounds, more than 60 single Chinese herbs and 30 active ingredients have good lipid-lowering and liver protection effects [17]. Chaihu-Shugan-San was recorded in the ancient masterpiece "Jing-Yue book" as the main treatment for liver depression symptoms. Modern pharmacology has revealed that Chaihu-Shugan-San provides liver protection and has anti-inflammatory properties. Zhang Liangdeng et al. [18] conducted 10 randomized controlled trials (RCTs) with 802 NAFLD subjects, and a meta-analysis showed that the total cholesterol, low-density lipoprotein, alanine aminotransferase (ALT), and aspartate aminotransferase (AST) levels decreased compared with those in a control group following Chaihu-Shugan-San treatment. In addition, a study showed that [19] Chaihu-Shugan-San could improve clinical symptoms and liver function in patients with digestive and metabolic diseases with liver qi stagnation and spleen deficiency syndromes. In an experimental study, preliminary results showed that $[20,21]$ Chaihu-ShuganSan could repress the inflammatory response and therefore alleviate the progression of NAFLD disease. Therefore, due to the close link between the liver and intestines, our study investigated the effects of Chaihu-Shugan-San on the gut microbiota, SCFA production and its impact on serum and liver lipids, serum LPS, inflammatory markers, and NLRP3 pathways in NAFLD rats.

\section{Materials and Methods}

2.1. Animals and Study Design. Twenty-four healthy male SD rats (SPF grade) were purchased from the Experimental Animal Center of Guangzhou University of Traditional Chinese Medicine (license number: SCXK (Guangdong) 2013-0034). After adaptive feeding for one week, all the rats were randomly divided into three groups $(n=8)$ : the normal control group (NC group), high-fat diet-fed group (HFD group), and Chaihu-Shugan-San decoction intervention group $(\mathrm{CH}$ group). Standard feed and the HFD were derived from the Guangdong Provincial Medical Laboratory Animal Center (license number: SCXK (Guangdong) 2013-0002) using the following HFD processing formula: $83 \%$ basal feed $+10 \%$ lard oil $+5 \%$ sucrose $+1.5 \%$ cholesterol $+0.5 \%$ cholate. The NC group rats were fed the standard diet, the HFD group rats were fed the $\mathrm{HFD}$, and the $\mathrm{CH}$ group rats were fed the HFD + Chaihu-Shugan-San $\left(9.6 \mathrm{~g} \cdot \mathrm{kg}^{-1} \cdot \mathrm{d}^{-1}\right)$ for 16 weeks. Experimental herbal doses were determined using the textbook Prescriptions of Chinese Materia Medica (tenth edition) [22] and preliminary research results [23]. All rats from each group had free access to water and were kept in an SPF animal laboratory with a dark and light cycle of 12 hours and temperature range of $18^{\circ} \mathrm{C}-22^{\circ} \mathrm{C}$.

2.2. Chaihu-Shugan-San Decoction Preparation. All ChaihuShugan-San formula granules used in this study were obtained from the same place and the same batch. According to the analysis method described in China Pharmacopoeia (2015 edition), the Chaihu-Shugan-San formula granules conformed to the China Pharmacopoeia standards (Committee, 2010). The TCM formula Chaihu-Shugan-San decoction consists of seven herbs, namely, Bupleurum scorzonerifolium Willd., Ligusticum chuanxiong Hort., Citrus aurantium L., Citrus reticulata Blanco, Paeonia lactiflora Pall., Cyperus rotundus L., and Glycyrrhiza uralensis Fisch., which were purchased from Jiangyin Tianjiang Pharmaceutical Co., Ltd. (Jiangsu, China), and the amounts of each herb in one unit of Chaihu-Shugan-San formula in each group are shown in Table 1. According to the amounts of each herb required for one unit of Chaihu-Shugan-San formula, Bupleurum scorzonerifolium Willd. (6 g), Ligusticum chuanxiong Hort. (5 g), Citrus aurantium L. (5 g), Citrus reticulata Blanco (6 
Table 1: The Chaihu-Shugan-San decoction of each dose.

\begin{tabular}{|c|c|c|c|c|}
\hline Chinese name & Common name & Botanical name & Part used & Weight (g) \\
\hline Chaihu & Bupleuri Radix & $\begin{array}{c}\text { Bupleurum } \\
\text { scorzonerifolium Willd. }\end{array}$ & Root & 6 \\
\hline Chuanxiong & Chuanxiong Rhizoma & $\begin{array}{c}\text { Ligusticum chuanxiong } \\
\text { Hort. }\end{array}$ & Rhizome & 5 \\
\hline Zhiqiao & Aurantii Fructus & Citrus aurantium L. & Fruit & 5 \\
\hline Chenpi & $\begin{array}{l}\text { Citri Reticulatae } \\
\text { pericarpium }\end{array}$ & Citrus reticulata Blanco & Pericarp & 6 \\
\hline Baishao & Paeoniae Radix Alba & Paeonia lactiflora Pall. & Root & 5 \\
\hline Xiangfu & Cyperi Rhizoma & Cyperus rotundus L. & Rhizome & 5 \\
\hline Zhigancao & $\begin{array}{c}\text { Glycyrrhizae Radix et } \\
\text { Rhizoma }\end{array}$ & $\begin{array}{l}\text { Glycyrrhiza uralensis } \\
\text { Fisch., Glycyrrhiza } \\
\text { inflata Bat. or } \\
\text { Glycyrrhiza glabra L. }\end{array}$ & Root and rhizome & 3 \\
\hline
\end{tabular}

g), Paeonia lactiflora Pall. (5 g), Cyperus rotundus L. (5 g), and Glycyrrhiza uralensis Fisch. (3 g) were weighed and then fully dissolved in $2500 \mathrm{~mL}$ of ultrapure water heated in a water bath as the stock solution. Then, $14 \mathrm{~mL}$ of methanol were added to $10 \mathrm{~mL}$ of stock solution, and ultrasonic treatment was conducted for $30 \mathrm{~min}$. Then, the solution was filtered with a $0.25-\mu \mathrm{m}$ microporous membrane at room temperature.

2.3. HPLC Analysis of the Major Components in the ChaihuShugan-San Decoction. To confirm the major components of the Chaihu-Shugan-San decoction used in this study, high-performance liquid chromatography (HPLC) analysis was performed. Briefly, chromatographic separation was performed on a COSMOSIL Packed Column 5C18-MS-II (4.6 $\mathrm{mm} \times 250 \mathrm{~mm}$ ) using acetonitrile as the mobile phase A, trifluoroacetic acid (TFA) as the mobile phase $\mathrm{B}$, and detection wavelengths of 210 and $235 \mathrm{~nm}$, respectively. Saikosaponin A was detected at a wavelength of $210 \mathrm{~nm}$, and ferulic acid, neohesperidin, naringin, hesperidin, paeoniflorin, liquiritin, and glycyrrhizic acid were detected at $235 \mathrm{~nm}$.

2.4. EchoMRI ${ }^{\mathrm{TM}}$ Analysis of Whole-Body Composition. Total body fat, lean mass, free water, and total body water content in vivo were evaluated using an EchoMRI 2012 (EchoMRI, Houston, TX, USA) and quantitative magnetic resonance body composition analyzers. These tests were conducted at week 16 prior to sacrifice as previously described [24].

2.5. Biochemical Measurements of Lipids and Inflammatory Markers in Serum and Liver Tissue. Blood plasma (3-5 mL) was centrifuged at $3000 \mathrm{rpm}$ and $4^{\circ} \mathrm{C}$ for $10 \mathrm{~min}$, and the upper layer of the serum was collected in a 1.5-mL EP tube. Hepatic tissue $(0.1 \mathrm{~g})$ was placed in isopropanol $(0.9 \mathrm{~mL})$ and homogenized with a tissuelyser-II homogenizer, followed by centrifugation at $3500 \mathrm{rpm}$ and $4^{\circ} \mathrm{C}$ for $10 \mathrm{~min}$. The clear supernatants were collected, and the levels of TC and TG in serum and liver tissue were determined with an automatic biochemical analyzer. Serum and liver TNF- $\alpha$, IL- $1 \beta$, and IL18 levels were quantitatively detected using an enzyme-linked immunosorbent assay. Serum LPS in the portal vein was determined with an endpoint chromogenic assay (No.160525, Xiamen Bioendo Technology, Co., Ltd., Fujian, China).

2.6. Western Blot Analyses. Approximately $200 \mathrm{mg}$ of liver tissue was added to $2 \mathrm{~mL}$ of RIPA lysate and $20 \mu \mathrm{L}$ of protease inhibitor. The ultrasonic homogenizer was used to homogenize the sample in an ice bath until no granules were detected. After centrifugation, the middle layer of the liquid was collected (the upper layer was oil, and the lower layer had a little precipitation), and centrifugation was repeated one time. For the BCA method and protein hyperthermia degeneration, the cells were transferred to a PVDF membrane and 5\% skim milk powder at room temperature for $1.5 \mathrm{~h}$. The membrane was immersed in the TLR4 antibody 1:500, NLRP3 antibody 1:500, ASC antibody 1:300, Caspase- 1 antibody 1:500, or NF- $\kappa$ B p65 antibody 1:1000 at $4^{\circ} \mathrm{C}$ overnight. After washing the membrane, goat anti-rabbit IgG $(\mathrm{H}+\mathrm{L})$, mouse/human ads-HRP (1:20,000; Southern Biotech, 4050-05), or rabbit anti-mouse IgG (H+L)$\operatorname{HRP}$ (1:10000; Southern Biotech, 6170-05) was incubated with the membrane for $1.5 \mathrm{~h}$ at room temperature. After washing the film, ECL luminescence solution was added, and the film was developed. The semiquantitative analysis of the bands was performed using software. The OD value of the target protein was divided by the OD value of the $\beta$-actin band as the final result.

2.7. Sequencing Analyses of Intestinal Microbiota. At 1-2 d before euthanasia, fresh fecal samples were collected under anaerobic conditions and immediately frozen in liquid nitrogen at the end of the 16-week intervention period. The genomic DNA of each fecal sample was extracted with QIAamp DNA mini kits (Qiagen, Valencia, CA, USA), and bacterial genome DNA was detected by gel electrophoresis. The sample DNA was amplified to enrich the V3-V4 region of $16 \mathrm{~S}$ rDNA genes in bacteria with a specific primer containing a barcode sequence [25]. Then, the PCR amplification product was recovered and quantified using a QuantiFluor ${ }^{\mathrm{TM}}$ fluorometer. The purified amplification products were mixed in equal amounts and connected through sequencing joints. 

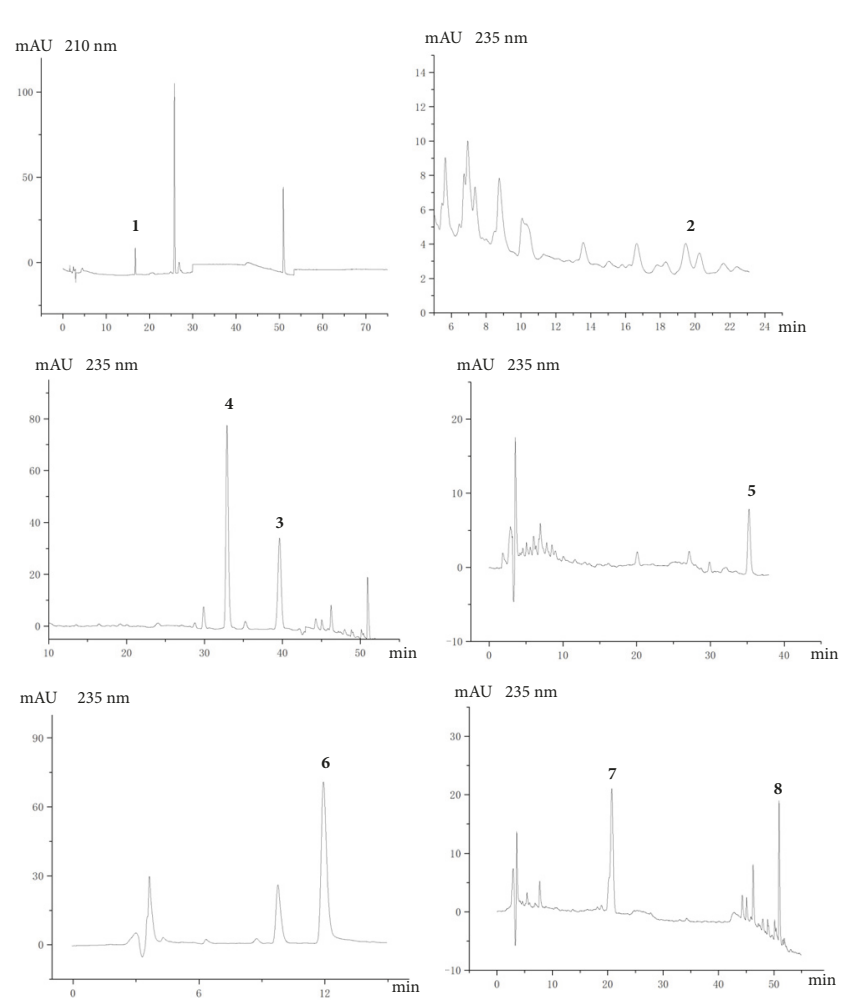

(a)

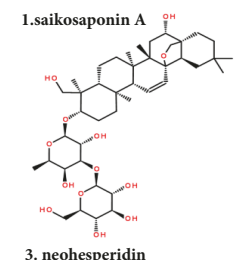

3. neohesperidi
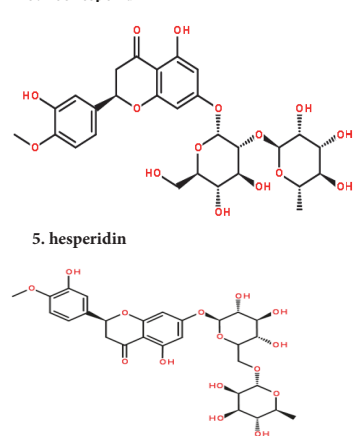

7.Liquiritin

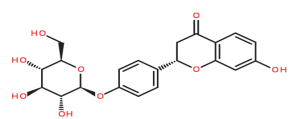

(b)

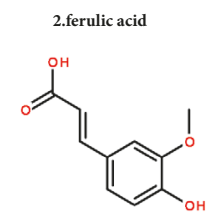

4.naringin
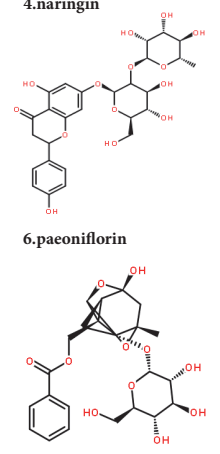

8.glycyrrhizic acid

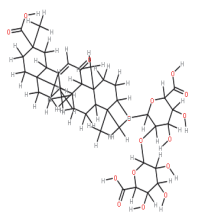

Figure 1: The herbal formula Chaihu-Shugan-San. (a) HPLC graphs of the Chaihu-Shugan-San decoction. High-performance liquid chromatography (HPLC) was performed to identify the phytochemical profiles of the Chaihu-Shugan-San decoction. (b) The main chemicals in the Chaihu-Shugan-San decoction were the following: 1, saikosaponin A; 2, ferulic acid; 3, neohesperidin; 4, naringin; 5, hesperidin; 6, paeoniflorin; 7, liquiritin; and 8, glycyrrhizic acid.

Then, a sequencing library was built, and sequencing with Hiseq2500 PE250 was conducted.

2.8. Gas Chromatography Analysis of Short-Chain Fatty Acids. At the end of the intervention period for 16 weeks, the rats were deprived of food for $12 \mathrm{~h}$, weighed, and injected with $2 \%$ sodium pentobarbital solution $(8 \mathrm{mg} / \mathrm{kg})$. The contents in the large intestine were frozen immediately after collection at the end of treatment and stored in liquid nitrogen until used. Standard reference materials for acetic acid, propionic acid, isobutyric acid, butyric acid, isovaleric acid, valeric acid, and 2-ethylbutyric acid (chromatographic grade) were obtained from the Aladdin Company in China. The $5 \mathrm{M}$ HCL, diethyl ether, and methanol (analytical grade) were purchased from Guangzhou Biological Technology Co., Ltd. (Guangdong, China). Gas chromatography (GC) was carried out for analysis of stool extracts as previously described [20]. Briefly, one gram of flesh-frozen stool samples was extracted with at least $5 \mathrm{~mL}$ of deionized water, mixed for approximately $5 \mathrm{~min}$, and brought up to a final suspension concentration of $17 \%(\mathrm{w} / \mathrm{w})$. The 2-methylbutanoic acid stock solution $(200 \mathrm{mM}, 25 \mu \mathrm{l})$ was added to the suspension at a final concentration of $1 \mathrm{mM}$. The tube was centrifuged for $5 \mathrm{~min}$ in a tabletop centrifuge, and the supernatant was collected and mixed with $5 \mathrm{M}$ HCL to adjust the $\mathrm{pH}$ value to $2-3$. The hexane layer was placed in a GC vial, and the vial was then capped and stored at $-20^{\circ} \mathrm{C}$ until GC analysis.
2.9. Statistical Analyses. Statistical analyses were performed using SPSS 19.0. Data are expressed as the mean \pm SEM. Differences were analyzed by one-way ANOVA and adjusted by Bonferroni correction to counteract the problem of multiple comparisons. $P$ values $<0.05$ were considered significant.

\section{Results}

3.1. The Major Components of the Chaihu-Shugan-San Decoction. The Chaihu-Shugan-San decoction containing Bupleurum scorzonerifolium Willd., Ligusticum chuanxiong Hort., Citrus aurantium L., Citrus reticulata Blanco, Paeonia lactiflora Pall., Cyperus rotundus L., and Glycyrrhiza uralensis Fisch. were prepared using the procedure in Section 2.2. The sample solution was prepared separately, and HPLC conditions were measured for eight components using the procedure in Section 2.3. The results are shown in Figure 1.

3.2. The Chaihu-Shugan-San Decoction Modulates the Intestinal Microbiota in NAFLD Rats. Based on the relative abundance of operational taxonomic units (OTUs), principal component analysis (PCA) showed that the sets of fecal samples in the NC, HFD, and $\mathrm{CH}$ groups are clearly separated, which indicates that the structures of the intestinal microbiota in these three groups were partially different (Figure 2). Furthermore, the relative abundance of family taxa had a $P$ value of $<0.05$ according to the differential expression analysis 


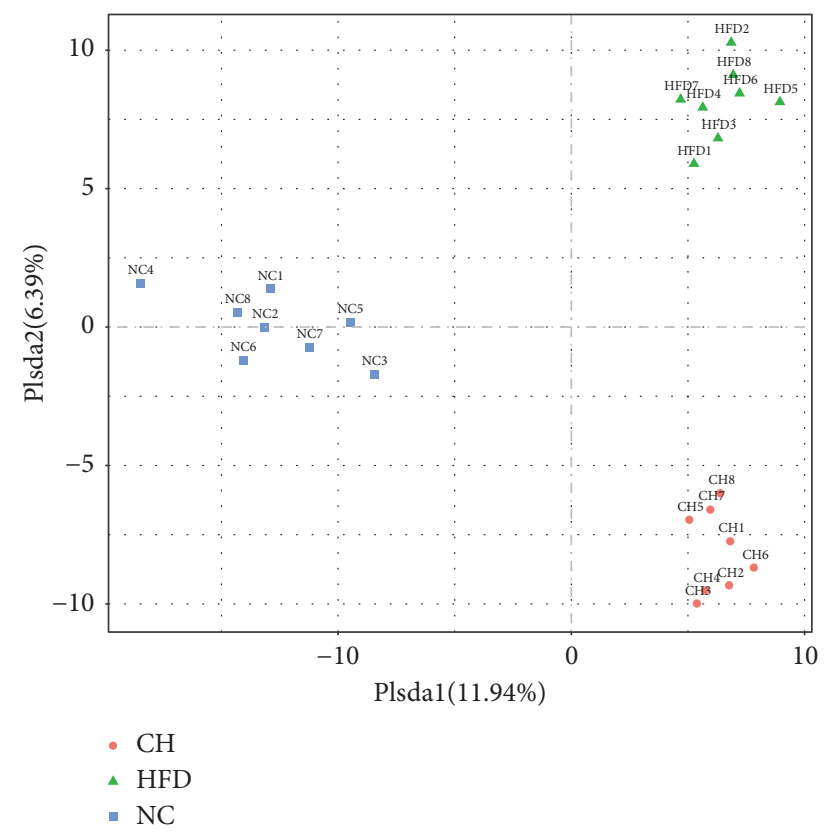

FIGURE 2: OTU principal component analysis (PCA). The vertical and ordinate are the first and second principal components, and the percentage in parentheses indicates the contribution of the principal component to the sample difference. The points on the graph indicate the individual samples. Different colors represent samples belonging to different groups.

(nonparametric ANOVA with false discovery rate [FDR] correction), which was expressed as a heat map (Figure 3), including hierarchical clustering (HCN). At the family level, the abundances of 44 strains in the HFD group were different compared with those in the NC group, especially for F16, Lactobacillaceae, Clostridiaceae, and Enterobacteriaceae $(P$ $<0.05)$. Compared with the HFD group, 18 Bacteriaceae in the $\mathrm{CH}$ group were different, in particular the abundances of Enterobacteriaceae $(0.375 \%$ versus $0.064 \%, P<0.05)$ and Staphylococcaceae $(0.049 \%$ versus $0.016 \%, P<0.05)$ were significantly different (Figures $4(\mathrm{a})$ and $4(\mathrm{~b})$ ). At the genus level, compared with the NC group, the abundances of 55 strains in the HFD group were different, especially Veillonella, Anaeroplasma and Cupriavidus $(P<0.05)$. Compared with the HFD group, the abundance of 27 strains, such as Veillonella $(0.096 \%$ versus $0.009 \%, P<0.01)$ and Anaeroplasma $(0.0005 \%$ versus $0.0178 \%, P<0.01)$, significantly changed $(P$ $<0.05$ ) (Figures 4(c) and 4(d)). Furthermore, compared with the NC group, the isobutyric acid and isovaleric acid levels in feces of the HFD group rats reduced by $35.6 \%$ and $34.5 \%$, respectively $(P<0.01)$. While compared with the HFD group, the butyric acid showed a tendency towards increase $(P=$ $0.074)$. But the differences of other SCFA in dry feces were not significant between the three groups (Table 2).

\subsection{The Chaihu-Shugan-San Decoction Reduced Bodyweight} and Total Body Fat Content in NAFLD Rats. Furthermore, body composition was assessed by EchoMRI analysis. Compared with the NC group, the body weight (g) and total body fat mass (g) of the HFD group significantly increased $(P<$
0.01). These indices of the $\mathrm{CP}$ group were all significantly lower than those of the HFD group $(P<0.05$ or $P<0.01)$. The results showed that Chaihu-Shugan-San significantly reduced HFD-induced weight gain and body fat accumulation (Figure 5, Supplemental Table 1).

3.4. The Chaihu-Shugan-San Decoction Reduced Lipid Accumulation and Chronic Low-Grade Inflammation in NAFLD Rats. Compared with the NC group, concentrations of TC, TG, TNF- $\alpha$, IL-1 $\beta$, and IL-18 in the serum and liver tissue homogenates of the HFD group significantly increased ( $P$ $<0.05$ or $P<0.01)$. In addition to serum TC, all of the above indicators significantly decreased $(P<0.05$ or $P<0.01)$. The results indicated the presence of severe lipid deposition and metabolic inflammation and that Chaihu-Shugan-San reduced the levels of liver fat and inflammatory cytokines in NAFLD rats (Figure 6, Supplemental Table 2).

3.5. Chaihu-Shugan-San Decoction Influenced the Protein Expression of the NLRP3 Pathway in NAFLD Rats. Compared with the NC group, the serum LPS levels and the expression of TLR4, NLRP3, ASC, Caspase-1, and NF-kB p65 protein in the HFD group significantly increased $(P<0.05$ or $P$ $<0.01)$. Compared with the HFD group, serum LPS and hepatic NLRP3, ASC, and Caspase-1 were lowered by ChaihuShugan-San $(P<0.05$ or $P<0.01)$ (Figure 7 , Supplemental Table 3).

\section{Discussion}

The Chinese herbal compound Chaihu-Shugan-San is composed of Bupleurum scorzonerifolium Willd., Ligusticum chuanxiong Hort., Citrus aurantium L., Citrus reticulata Blanco, Paeonia lactiflora Pall., Cyperus rotundus L., and Glycyrrhiza uralensis Fisch.. Chaihu-Shugan-San has a wide range of clinical applications, especially for the treatment of depression [26], hyperlipidemia, NAFLD [27], and some digestive diseases [28]. The efficacy of the Chinese herbal compound was determined according to the composition and content of the compound. In our experiments, the chemical compositions of Bupleurum scorzonerifolium Willd., Ligusticum chuanxiong Hort., Citrus aurantium L., Citrus reticulata Blanco, Paeonia lactiflora Pall., Cyperus rotundus L., and Glycyrrhiza uralensis Fisch. extracts were analyzed by HPLC. The results showed that the fingerprints, including saikosaponin A, ferulic acid, neohesperidin, naringin, hesperidin, paeoniflorin, liquiritin, and glycyrrhizic acid, were consistent with the reference substance. Modern pharmacodynamics also revealed that Chaihu-Shugan-San contains a variety of chemical components, which mainly include saponins, flavonoids, terpenoids, and phenolic acids. These ingredients were directly related to the many pharmacological effects of the compound. It was found that saikosaponin A and saikosaponin D have anti-inflammatory effects [29-31] and regulate lipid metabolism [32]. Li R. believed that saikosaponin could inhibit the release of inflammatory mediators and granuloma growth and inhibit a 5-HT-induced capillary permeability increase, as well as significantly inhibiting leukocyte migration and connective tissue hyperplasia [33]. 


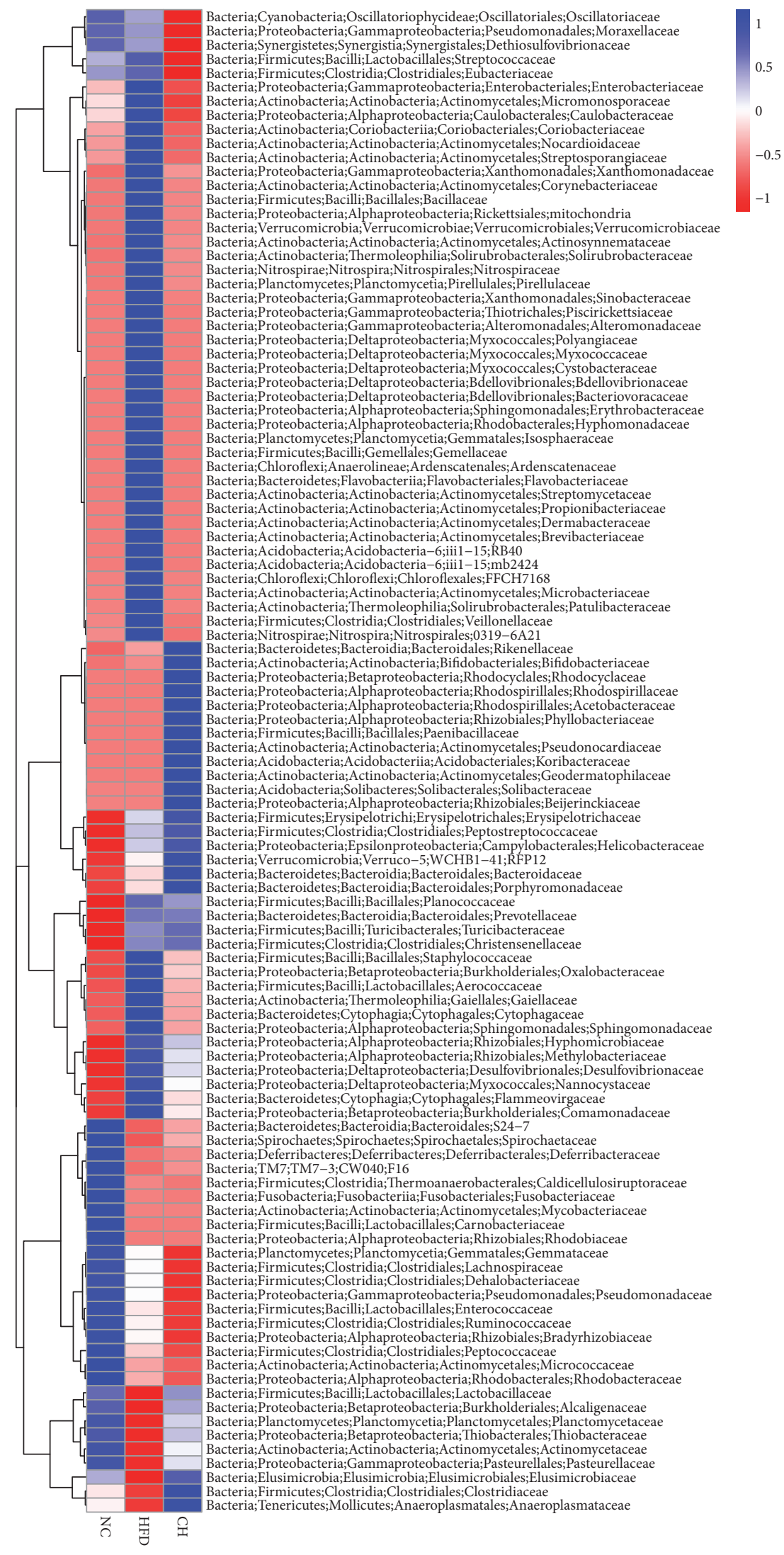

FIGURE 3: Heat map of bacteria with changes between each two groups at the family level. Hierarchical clustering with a heat map shows the relative abundance of representative OTUs (i.e., samples with the greatest difference among the three group means from each family) selected due to differences corresponding to $P<0.05$, obtained with differential expression analysis of the three groups. The OTUs are shown as phylum, class, order, and family. 


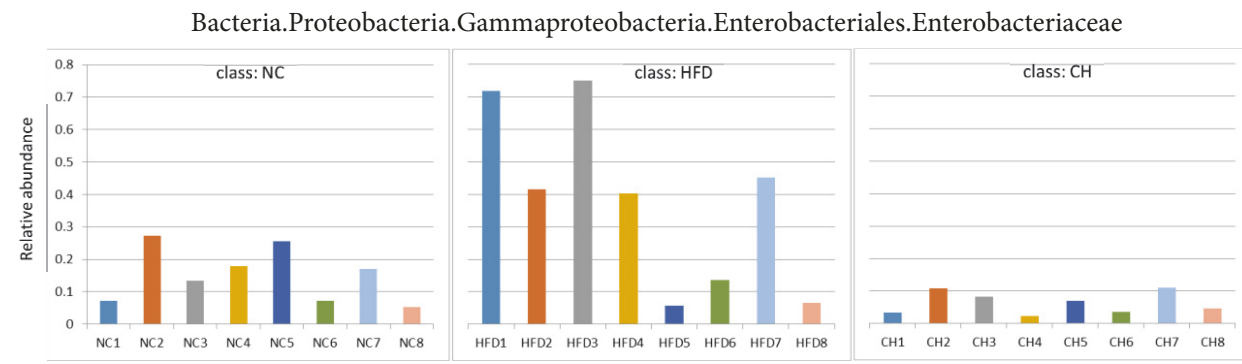

(a)

Bacteria.Firmicutes.Bacilli.Bacillales.Staphylococcaceae

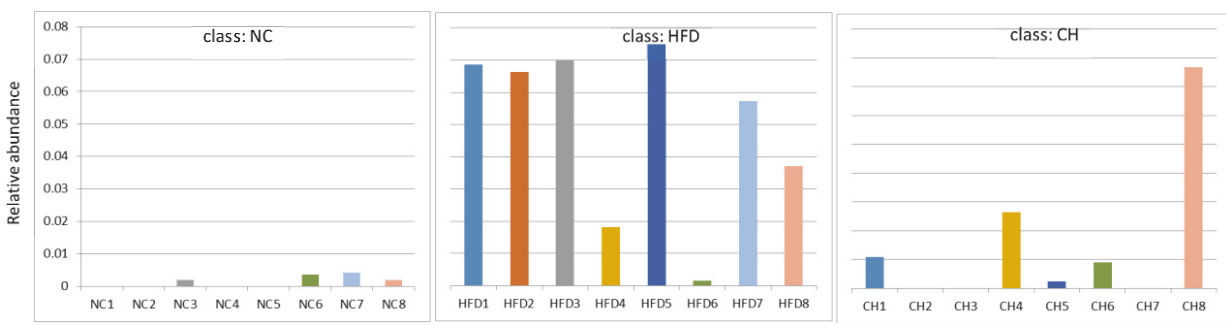

(b)

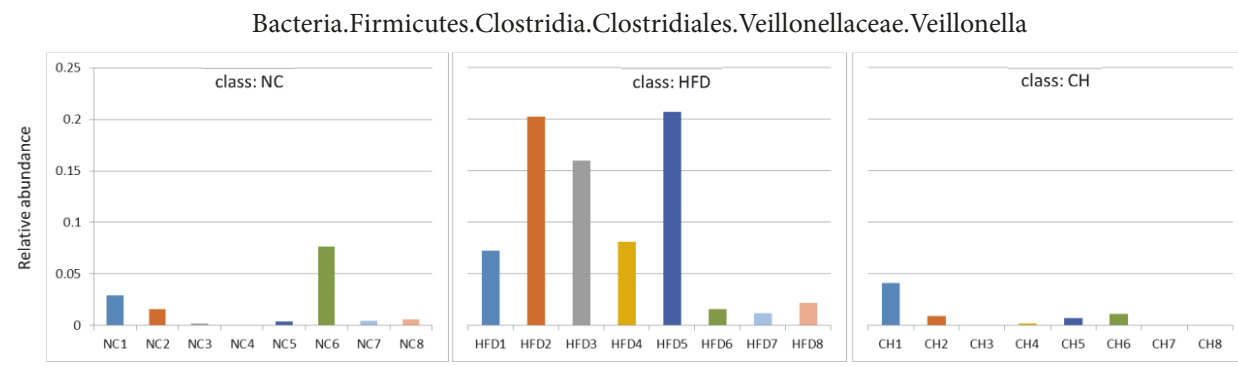

(c)

Bacteria.Tenericutes.Mollicutes.Anaeroplasmatales.Anaeroplasmataceae.Anaeroplasma
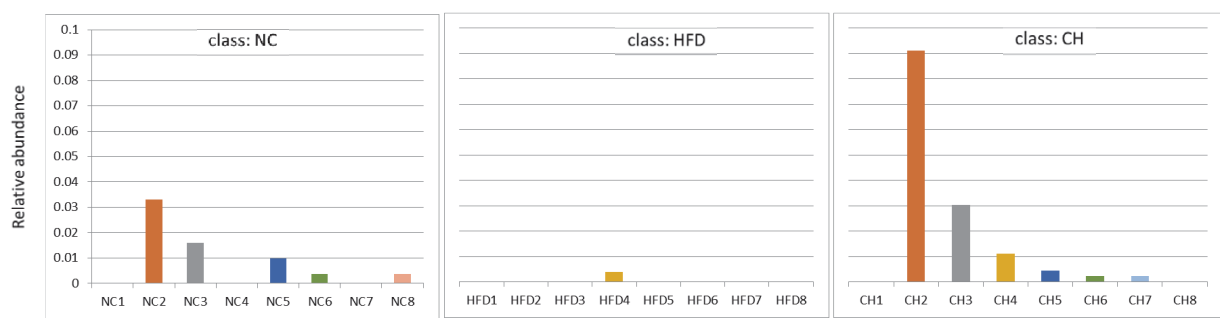

(d)

FIGURE 4: The actual representation of individual species in the comparison group NC, HFD, and CH. (a) Enterobacteriaceae, (b) Staphylococcaceae, (c) Veillonella, and (d) Anaeroplasma, $n=8$.
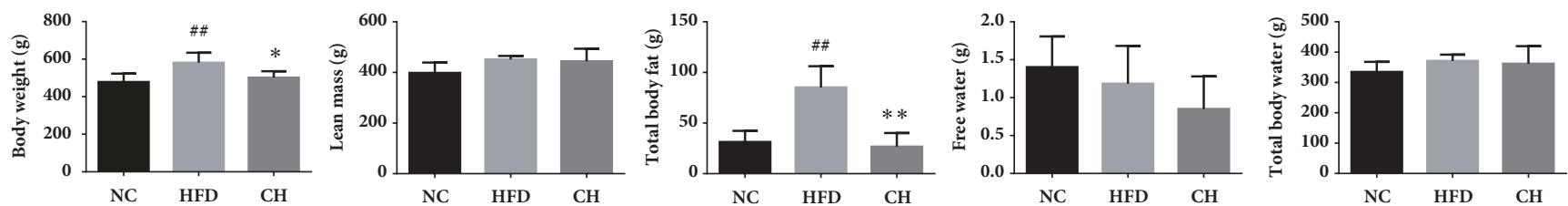

FIGURE 5: Body weight, total body fat, lean mass, free water, and total body water in three SD rat groups fed a control diet or a HFD supplemented with Chaihu-Shugan-San for 16 weeks. ${ }^{\#} P<0.05 \mathrm{NC}$ versus $\mathrm{HFD},{ }^{\# \#} P<0.01 \mathrm{NC}$ versus $\mathrm{HFD},{ }^{*} P<0.05 \mathrm{HFD}$ versus $\mathrm{CH}$, ${ }^{* *} P<0.01 \mathrm{HFD}$ versus $\mathrm{CH}, \mathrm{n}=6-8$. 

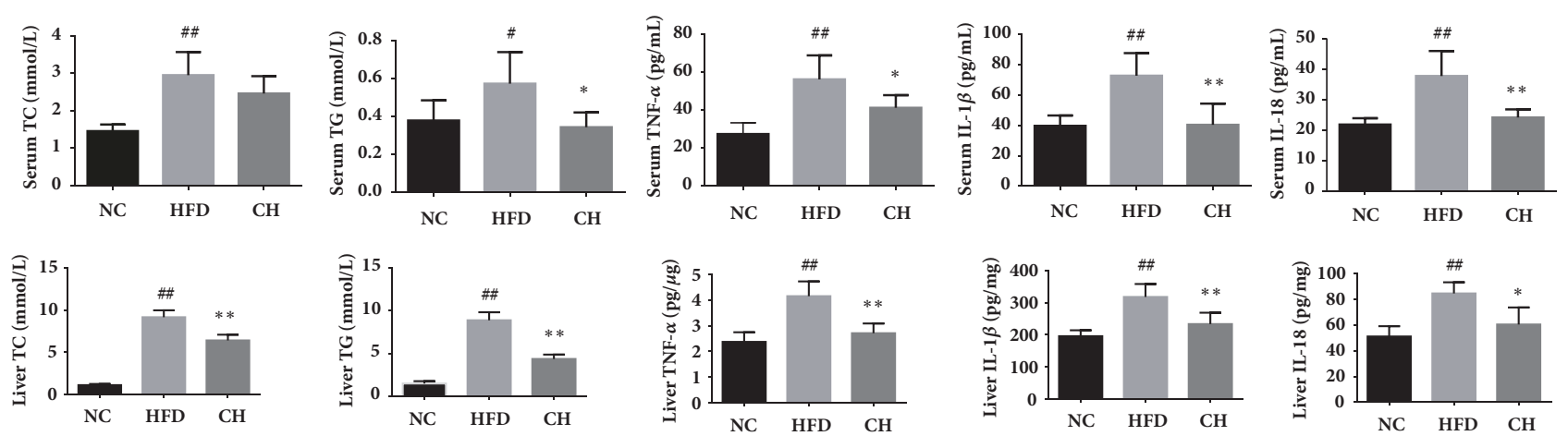

FIGURE 6: Serum and liver TC, TG, TNF- $\alpha$, IL-1 $\beta$, and IL-18 in three groups: SD rats fed a control diet or a HFD supplemented with ChaihuShugan-San for 16 weeks. ${ }^{\#} P<0.05$ NC versus HFD, ${ }^{\# \#} P<0.01$ NC versus HFD, ${ }^{*} P<0.05$ HFD versus $\mathrm{CH}$, and ${ }^{* *} P<0.01 \mathrm{HFD}$ versus $\mathrm{CH}$, $\mathrm{n}=6$.

TABLE 2: Mean SCFA (mmol/L) concentrations in dry feces of experimented rats.

\begin{tabular}{lcccccc}
\hline Category & acetic acid & propionic acid & isobutyric acid & butyric acid & isovaleric acid & valeric acid \\
\hline NC group & $0.269 \pm 0.116$ & $0.241 \pm 0.052$ & $0.045 \pm 0.011$ & $0.438 \pm 0.106$ & $0.087 \pm 0.014$ & $0.064 \pm 0.009$ \\
HFD group & $0.294 \pm 0.105$ & $0.336 \pm 0.112$ & $0.029 \pm 0.009^{\# \#}$ & $0.373 \pm 0.151$ & $0.057 \pm 0.009$ & $0.048 \pm 0.025$ \\
CH group & $0.389 \pm 0.183$ & $0.352 \pm 0.119$ & $0.032 \pm 0.008$ & $0.393 \pm 0.114$ & $0.044 \pm 0.016$ & $0.047 \pm 0.008$ \\
\hline
\end{tabular}

\#\# Significant difference compared with NC group rats in fecal SCFAs based on ANOVA as described in the Methods. $P<0.01 \mathrm{NC}$ versus HFD, $\mathrm{n}=8$.

Tetramethylpyrazine, which is the active component of Ligusticum chuanxiong Hort., has anti-inflammatory effects [34], whereas ferulic acid regulates lipid metabolism by inhibiting lipid oxidation and lowering cholesterol levels in blood lipids [35]. Hesperidin, naringin, and paeoniflorin, which are the active ingredients of Citrus aurantium L. and Paeonia lactiflora Pall., also have anti-inflammatory effects [36-38] and regulate lipid metabolism $[39,40]$. The study by Wang W. et al. [41] showed that hesperidin may inhibit the expression and activity of COX-2 in liver tissue, and, therefore, it had a therapeutic effect on NAFLD rats. It could be seen that the active ingredients of Chaihu-Shugan-San had obvious antiinflammatory, antioxidative, and lipid metabolism regulatory effects, but the exact target of this compound needs to be further studied.

It has been considered that the intestinal microbiota produces some toxic components that may potentially harm the liver. These bacterial toxic components reach the liver through the portal vein, activate liver Kupffer cells, and then stimulate the production of NO and cytokines, which is a process known as intestinal endotoxemia. Production of endogenous ethanol and LPS could induce intestinal macrophage activation, which stimulates the secretion of proinflammatory cytokines and may be one of the possible intestinal microbiota mechanisms affecting intestinal barrier function [42]. In all bacterial products, LPS appeared to be the most important in the pathogenesis of NAFLD. LPS is the active ingredient of endotoxins, especially when it is combined with lipopolysaccharide-binding protein (LBP), CD14, TLR4, and lymphocyte antigen 96. Endogenous LPS is continuously produced by the death of intestinal gramnegative bacteria and then migrates into the liver through TLR4-mediated signaling pathways. Therefore, changes in intestinal permeability could promote endotoxins in the liver.
It has been found that TLR4 works primarily in a MyD88dependent pathway. TLR4 binds to CD14 on the surface of cell membranes, initiates LPS-induced signal transduction, and activates NF- $\kappa \mathrm{B}$ and the downstream proinflammatory cytokines TNF and cyclooxygenase 2[43]. A study was conducted to establish a model of fatty liver in rats using intravenous nutrition, and polymyxin was administered to inhibit the activity of endogenous LPS. The results showed that TNF production, liver fat, and TG deposition were remarkably reduced by polymyxin [44]. Our study revealed that, compared with the NC group, the expression of TLR4 and NF- $\kappa \mathrm{B}$ p65 in the liver tissue of the HFD group significantly increased $(P<0.05)$, whereas the Chaihu-ShuganSan mildly counteracts these effects. Accompanied by an increase in serum LPS, this increase showed that HFD promotes intestinal microbiota imbalance and increasingly severe intestinal endotoxemia. Furthermore, LPS may induce TLR4 pathway-related protein activation and the onset of NAFLD.

As previously mentioned, activation of the NLRP3 inflammasome is considered to be a key link in TLR4mediated hepatocyte injury, immune cell activation, and expansion of liver inflammation in NAFLD [11]. The activation of the NLRP3 inflammasome and the release of downstream IL- $1 \beta$ and IL- 18 have been shown to be associated with the pathogenesis of various liver diseases [45]. Currently, the focus of this field is on the effects of dietary and intestinal microflora interactions on the activation of inflammatory mediators in the liver [46]. Another study found that mature IL-1 $\beta$ could enhance the immune signals of liver immune cells, which leads to upregulation of monocyte chemoattractant protein-1 (MCP-1) and TNF- $\alpha$ expression. MCP-1 aggravated hepatocellular steatosis, while IL-1 $\beta$ was more sensitive to TNF-induced hepatocellular toxicity. In addition, 

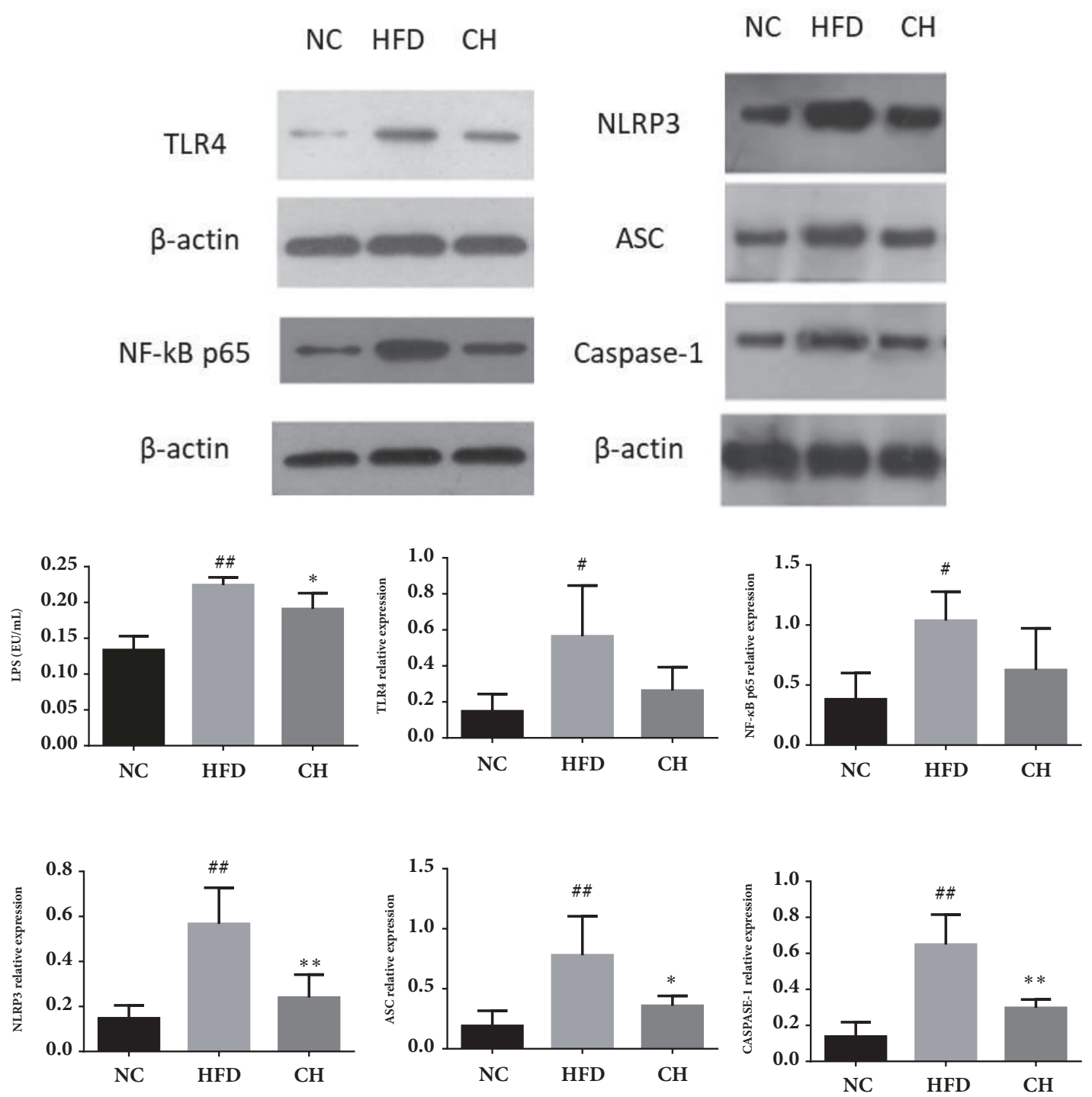

FIGURE 7: Serum LPS and protein expression of TLR4, NLRP3, ASC, Caspase-1, and NF-kB p65 in SD rats fed a control diet or a HFD supplemented with Chaihu-Shugan-San for 16 weeks. These proteins were quantified via western blotting in the hepatic tissue. ${ }^{\#} P<0.05 \mathrm{NC}$ versus HFD, ${ }^{\# \#} P<0.01 \mathrm{NC}$ versus HFD, ${ }^{*} P<0.05 \mathrm{HFD}$ versus $\mathrm{CH}$, and ${ }^{* *} P<0.01 \mathrm{HFD}$ versus $\mathrm{CH}, \mathrm{n}=4$.

the proinflammatory effects of IL-1 $\beta$ may be synergistic with TLR4 signaling and significantly enhance LPS-induced release of inflammatory cytokines [47]. In our study, we found increased expression of the NLRP3 inflammasome in NAFLD rats, and Chaihu-Shugan-San significantly inhibited overexpression of the NLRP3 inflammasome. This result suggested that NLRP3 may be a therapeutic target of ChaihuShugan-San.

Additionally, we found that the structure of the intestinal microflora in NAFLD rats was partially reconstructed in HFD-fed NAFLD rats, and the enrichment and diversity of intestinal microflora changed after Chaihu-Shugan-San treatment for 16 weeks. At the family level, we found that the abundance of Enterobacteriaceae decreased by nearly $90 \%$ in NAFLD rats $(P<0.05)$ compared with that in the NC group rats. Enterobacteriaceae is a major bacterium among the gram-negative bacilli, which include some conditional pathogens, such as Escherichia coli, Salmonella, Shigella, and Klebsiella, which are an important source of LPS. A recent RCT showed that aerobic bacteria, such as Enterococcus faecalis and Enterobacteriaceae, were significantly increased in the NASH group and that anaerobes, such as Bacillus bifidus and Lactobacillus, were reduced compared with the amount in the healthy group [48]. In this study, Chaihu-Shugan-San significantly reduced the abundance of Enterobacteriaceae and the concentration of serum LPS. It is likely that one of the intestinal mechanisms of Chaihu-Shugan-San alleviated intestinal endotoxemia in NAFLD rats. In addition, at the genus level, we were surprised to find that the abundance of Veillonella increased significantly in the HFD group rats, and Anaeroplasma decreased significantly. Currently, the Veillonella genus is believed to produce SCFAs through the succinic acid metabolic pathway, and the increase in the abundance of the Veillonella genus often leads to an increase 
in intestinal SCFA concentrations [49]. Compared with those in the NC group, this study found that isobutyric acid and isovaleric acid levels of dry feces in the HFD group were significantly reduced. But the differences of other SCFA in dry feces were not significant among the three groups. Therefore, the relationship between SCFAs and NAFLD needs further investigation.

\section{Conclusions}

In summary, the Chaihu-Shugan-San decoction modulated intestinal microbe dysbiosis, reduced body fat and intrahepatic fat accumulation and alleviated LPS-induced endotoxemia and inflammatory factor expression, which are all processes related to the NLRP3 inflammasome in NAFLD rats.

\section{Conflicts of Interest}

The authors declare that they have no conflicts of interest.

\section{Acknowledgments}

The authors thank Pinghe Yin, Yang Hu, and Huanyong Li from Jinan University Analytical and Testing Center for technical assistance and Guangzhou Genedenovo Biotechnology Co. Ltd., for providing the methods for partial bioinformatics analysis. The present work was supported by a grant from National Natural Science Foundation of China (nos. 81774165 and 81573844), Natural Science Foundation of Guangdong in China (no. 2016A030313824), Traditional Chinese Medicine Bureau of Guangdong in China (no. 20161065), and National Health and Family Planning Commission of Guangdong in China (no. A2016583, A2017228, A2017140).

\section{Supplementary Materials}

Supplemental Table 1: Body weight and body composition. (DOCX) Supplemental Table 2: Lipids and inflammatory cytokines in serum and liver. (DOCX) Supplemental Table 3: The relative expression of TLR4, NLRP3, ASC, Caspase-1, NF-kB p65. (DOCX) (Supplementary Materials)

\section{References}

[1] A. Wree, L. Broderick, A. Canbay, H. M. Hoffman, and A. E. Feldstein, "From NAFLD to NASH to cirrhosis-new insights into disease mechanisms," Nature Reviews Gastroenterology \& Hepatology, vol. 10, no. 11, pp. 627-636, 2013.

[2] S. Watanabe, E. Hashimoto, K. Ikejima et al., "Evidence-based clinical practice guidelines for nonalcoholic fatty liver disease/nonalcoholic steatohepatitis," Journal of Gastroenterology, vol. 50, no. 4, pp. 364-377, 2015.

[3] M. Rinella and M. Charlton, "The globalization of nonalcoholic fatty liver disease: Prevalence and impact on world health," Hepatology, vol. 64, no. 1, pp. 19-22, 2016.

[4] J.-G. Fan and G. C. Farrell, "Epidemiology of non-alcoholic fatty liver disease in China," Journal of Hepatology, vol. 50, no. 1, pp. 204-210, 2009.
[5] Z. M. Younossi, A. B. Koenig, D. Abdelatif, Y. Fazel, L. Henry, and M. Wymer, "Global epidemiology of nonalcoholic fatty liver disease-meta-analytic assessment of prevalence, incidence, and outcomes," Hepatology, vol. 64, no. 1, pp. 73-84, 2016.

[6] J. M. Clark, F. L. Brancati, and A. M. Diehl, "The prevalence and etiology of elevated aminotransferase levels in the United States," American Journal of Gastroenterology, vol. 98, no. 5, pp. 960-967, 2003.

[7] G. A. Michelotti, M. V. Machado, and A. M. Diehl, "NAFLD, NASH and liver cancer," Nature Reviews Gastroenterology \& Hepatology, vol. 10, no. 11, pp. 656-665, 2013.

[8] J. Ertle, A. Dechêne, J. P. Sowa et al., "Non-alcoholic fatty liver disease progresses to hepatocellular carcinoma in the absence of apparent cirrhosis," International Journal of Cancer, vol. 128, no. 10, pp. 2436-2443, 2011.

[9] J. D. Yang, B. Kim, S. O. Sanderson et al., "Hepatocellular carcinoma in Olmsted County, Minnesota, 1976-2008," Mayo Clinic Proceedings, vol. 87, no. 1, pp. 9-16, 2012.

[10] J. A. Marrero, R. J. Fontana, G. L. Su, H. S. Conjeevaram, D. M. Emick, and A. S. Lok, "NAFLD may be a common underlying liver disease in patients with hepatocellular carcinoma in the United States," Hepatology, vol. 36, no. 6, pp. 1349-1354, 2002.

[11] G. Szabo and J. Petrasek, "Inflammasome activation and function in liver disease," Nature Reviews Gastroenterology \& Hepatology, vol. 12, no. 7, pp. 387-400, 2015.

[12] X. Cao, "Self-regulation and cross-regulation of patternrecognition receptor signalling in health and disease," Nature Reviews Immunology, vol. 16, no. 1, pp. 35-50, 2016.

[13] A. A. Shanab, P. Scully, O. Crosbie et al., "Small intestinal bacterial overgrowth in nonalcoholic steatohepatitis: association with toll-like receptor 4 expression and plasma levels of interleukin 8," Digestive Diseases and Sciences, vol. 56, no. 5, pp. 1524-1534, 2011.

[14] L. Zhu, S. S. Baker, C. Gill et al., "Characterization of gut microbiomes in nonalcoholic steatohepatitis (NASH) patients: a connection between endogenous alcohol and NASH," Hepatology, vol. 57, no. 2, pp. 601-609, 2013.

[15] M. Mouzaki, E. M. Comelli, B. M. Arendt et al., "Intestinal microbiota in patients with nonalcoholic fatty liver disease," Hepatology, vol. 58, no. 1, pp. 120-127, 2013.

[16] M. D. Spencer, T. J. Hamp, R. W. Reid, L. M. Fischer, S. H. Zeisel, and A. A. Fodor, "Association between composition of the human gastrointestinal microbiome and development of fatty liver with choline deficiency," Gastroenterology, vol. 140, no. 3, pp. 976-986, 2011.

[17] C. P. Commission, Pharmacopoeia of the People's Republic of China 2015, China Medical Science and Technology Press, Beijing, China, 2015.

[18] L. D. Zhang, X. H. Sun, W. Wei, and K. W. Yao, "Systematic evaluation and meta analysis on nonalcoholic fatty liver disease treated with Chaihu Shugan San," World Journal of Integrated Traditional and Western Medicine, vol. 9, no. 9, pp. 1004-1007, 2014.

[19] R. W. Cheng, P. Li, and L. P. Deng, "Effect of TACE combined with Chaihu Shugan Powder on Hepatocellular Carcinoma of Liver Depression and Spleen Deficiency," Guiding Journal of Traditional Chinese Medicine and Pharmacy, vol. 22, no. 20, pp. 20-23, 2016.

[20] X. W. Gong, Y. J. Xu, Q. H. Yang et al., "Effects of soothing liver and invigorating spleen recipes on the IKK $\beta$-NF- $\kappa$ B Signaling pathway in kupffer cells of nonalcoholic steatohepatitis rats," 
Evidence-Based Complementary and Alternative Medicine, vol. 2015, Article ID 687690, 9 pages, 2015.

[21] Q.-H. Yang, Y.-J. Xu, Y.-Z. Liu et al., "Effects of Chaihu-ShuganSan and Shen-Ling-Bai-Zhu-San on p38 MAPK Pathway in Kupffer Cells of Nonalcoholic Steatohepatitis," Evidence-Based Complementary and Alternative Medicine, vol. 2014, Article ID 671013, 8 pages, 2014.

[22] J. Li and J. W. Lian, Pharmacology of Traditional Chinese Medical Formulae, Chinese Medicine Publishing House of China, 2016.

[23] X. W. Gong, Q. H. Yang, H. Z. Yan et al., "Effects of soothing liver and invigorating spleen recipes on LPS-induced hepatocytes injury of rats and TLR4/p38MAPK signal pathway," China Journal of Chinese Materia Medica, vol. 39, no. 20, pp. 40274033, 2014.

[24] M. Peyrou, L. Bourgoin, A.-L. Poher et al., "Hepatic PTEN deficiency improves muscle insulin sensitivity and decreases adiposity in mice," Journal of Hepatology, vol. 62, no. 2, pp. 421429, 2015.

[25] H. Siddiqui, A. J. Nederbragt, K. Lagesen, S. L. Jeansson, and K. S. Jakobsen, "Assessing diversity of the female urine microbiota by high throughput sequencing of $16 \mathrm{~S}$ rDNA amplicons," BMC Microbiology, vol. 11, article 244, 2011.

[26] Y. H. Chen and Y. Zhou, "Experimental study on pharmacology of the anti-depression effect of Chaihu-Shugan-San," Journal of Jiangxi University of TCM, vol. 16, no. 4, pp. 59-60, 2004.

[27] C. Pan and J. R. Huang, "Clinical observation on the treatment of 82 cases of non-alcoholic fatty liver disease," Lishizhen Medicine and Materia Medica Research, vol. 20, no. 8, pp. 20102011, 2009.

[28] S. Z. Guang, "A 60 cases of chronic erosive gastritis were treated by Chaihu-Shugan-San," Journal of Shanxi College of Traditional Chinese Medicine, vol. 30, no. 5, pp. 21-22, 2007.

[29] N.-R. Han, H.-M. Kim, and H.-J. Jeong, "Inactivation of cysteinaspartic acid protease (caspase)-1 by saikosaponin A," Biological \& Pharmaceutical Bulletin, vol. 34, no. 6, pp. 817-823, 2011.

[30] C. Lu, Z. Yuan, X. Zhang et al., "Saikosaponin a and its epimer saikosaponin $\mathrm{d}$ exhibit anti-inflammatory activity by suppressing activation of NF- $\kappa \mathrm{B}$ signaling pathway," International Immunopharmacology, vol. 14, no. 1, pp. 121-126, 2012.

[31] X. Ma, C. Dang, H. Kang et al., "Saikosaponin-D reduces cisplatin-induced nephrotoxicity by repressing ROS-mediated activation of MAPK and NF- $\kappa$ B signalling pathways," International Immunopharmacology, vol. 28, no. 1, pp. 399-408, 2015.

[32] Z. Wei, J. Wang, M. Shi, W. Liu, Z.-T. Yang, and Y. Fu, "Saikosaponin a inhibits LPS-induced inflammatory response by inducing liver $\mathrm{X}$ receptor alpha activation in primary mouse macrophages," Oncotarget, vol. 7, no. 31, pp. 48995-49007, 2016.

[33] R. G. Li, "Analysis of active ingredients and pharmacological action of Chaihu-Shugan-San," Journal of Shanxi College of Traditional Chinese Medicine, vol. 34, no. 6, pp. 750-751, 2013.

[34] X. Wu, F. Zhang, X. Xiong et al., "Tetramethylpyrazine reduces inflammation in liver fibrosis and inhibits inflammatory cytokine expression in hepatic stellate cells by modulating NLRP3 inflammasome pathway," IUBMB Life, vol. 67, no. 4, pp. 312-321, 2015.

[35] J. Naowaboot, P. Piyabhan, N. Munkong, W. Parklak, and P. Pannangpetch, "Ferulic acid improves lipid and glucose homeostasis in high-fat diet-induced obese mice," Clinical and Experimental Pharmacology and Physiology, vol. 43, no. 2, pp. 242-250, 2016.
[36] S. O. Rotimi, G. E. Bankole, I. B. Adelani, and O. A. Rotimi, "Hesperidin prevents lipopolysaccharide-induced endotoxicity in rats," Immunopharmacology and Immunotoxicology, vol. 38, no. 5, pp. 364-371, 2016.

[37] W. Cao, W. Zhang, and J. Liu, "Paeoniflorin improves survival in LPS-challenged mice through the suppression of TNF-alpha and IL-1beta release and augmentation of IL-10 production," International Immunopharmacology, vol. 11, no. 2, pp. 172-178, 2011.

[38] Z. Ma, L. Chu, H. Liu et al., "Paeoniflorin alleviates nonalcoholic steatohepatitis in rats: Involvement with the ROCK/ NF-kappaB pathway," International Immunopharmacology, vol. 38, pp. 377-384, 2016.

[39] A. M. Mahmoud, "Hesperidin protects against cyclophosphamide-induced hepatotoxicity by upregulation of ppar $\gamma$ and abrogation of oxidative stress and inflammation," Canadian Journal of Physiology and Pharmacology, vol. 92, no. 9, pp. 717724, 2014.

[40] L. J. Zhang, B. Yang, and B. P. Yu, "Paeoniflorin protects against nonalcoholic fatty liver disease induced by a high-fat diet in mice," Biological \& Pharmaceutical Bulletin, vol. 38, no. 7, pp. 1005-1011, 2015.

[41] W. Wang, J. Li, R. Li, L. Xiong, X. Y. Fan, and D. Y. Ren, "Effect of hesperidin on the expression and activity of COX-2 in rats with non-alcoholic fatty liver disease," Acta Universitatis Medicinalis Anhui, vol. 46, no. 9, pp. 897-901, 2011.

[42] S. F. Solga and A. M. Diehl, "Non-alcoholic fatty liver disease: lumen-liver interactions and possible role for probiotics," Journal of Hepatology, vol. 38, no. 5, pp. 681-687, 2003.

[43] G. L. Su, "Lipopolysaccharides in liver injury: molecular mechanisms of Kupffer cell activation," American Journal of Physiology-Gastrointestinal and Liver Physiology, vol. 283, no. 2, pp. G256-G265, 2002.

[44] I. Pappo, H. Bercovier, E. M. Berry, Y. Haviv, R. Gallily, and H. R. Freund, "Polymyxin B reduces total parenteral nutritionassociated hepatic steatosis by its antibacterial activity and by blocking deleterious effects of lipopolysaccharide," Journal of Parenteral and Enteral Nutrition, vol. 16, no. 6, pp. 529-532, 1992.

[45] G. Szabo and T. Csak, "Inflammasomes in liver diseases," Journal of Hepatology, vol. 57, no. 3, pp. 642-654, 2012.

[46] N. J. Wood, "Dysbiosis driven by inflammasome deficiency exacerbates hepatic steatosis and governs rate of NAFLD progression," Nature Reviews Gastroenterology \& Hepatology, vol. 9, no. 3, pp. 123-123, 2012.

[47] J. Petrasek, S. Bala, T. Csak et al., "IL-1 receptor antagonist ameliorates inflammasome-dependent alcoholic steatohepatitis in mice," The Journal of Clinical Investigation, vol. 122, no. 10, pp. 3476-3489, 2012.

[48] L. H. Yang, J. Cai, and D. F. Chen, "Alteration and significance of intestinal flora in patients with NASH," Journal of Clinical Hepatology, vol. 28, no. 2, pp. 124-126, 2012.

[49] P. Louis, G. L. Hold, and H. J. Flint, "The gut microbiota, bacterial metabolites and colorectal cancer," Nature Reviews Microbiology, vol. 12, no. 10, pp. 661-672, 2014. 


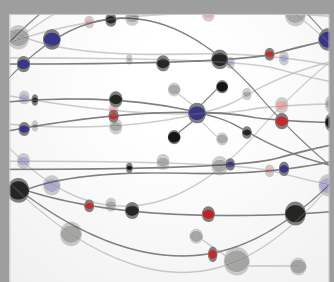

The Scientific World Journal
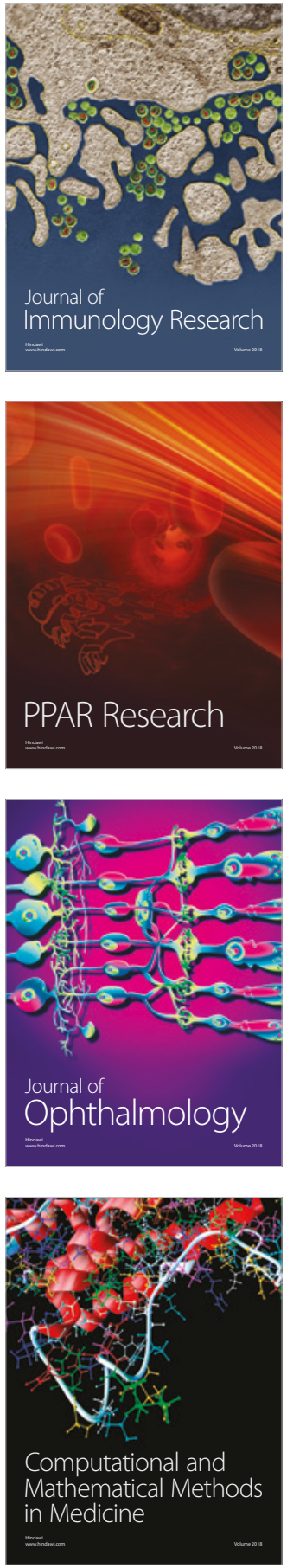

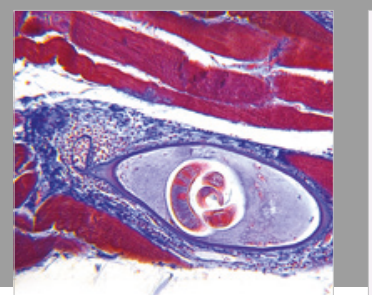

Gastroenterology Research and Practice

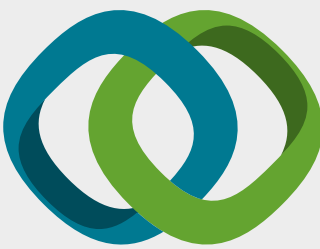

\section{Hindawi}

Submit your manuscripts at

www.hindawi.com
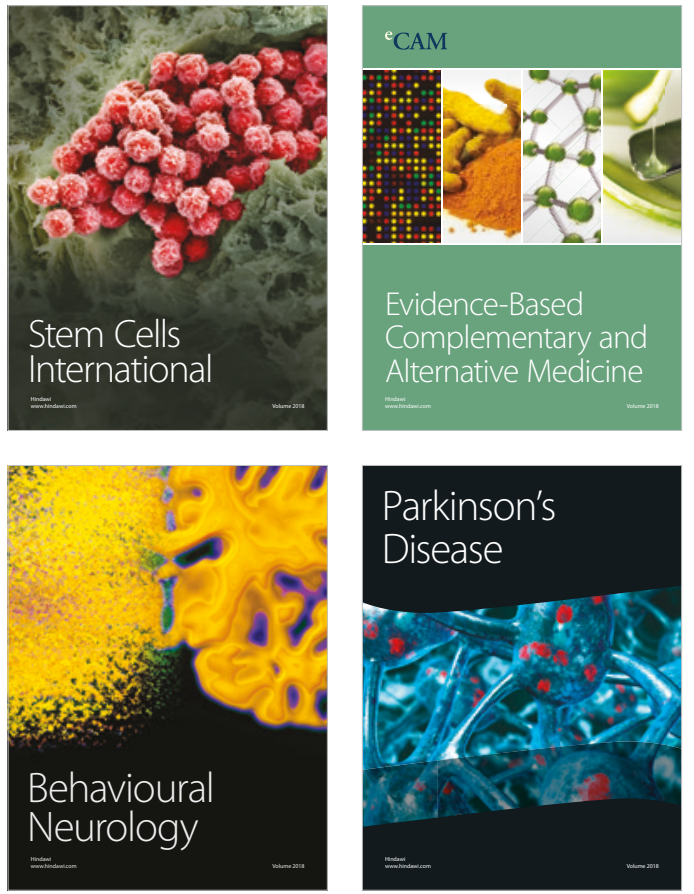

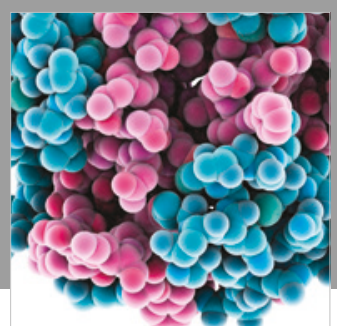

ournal of

Diabetes Research

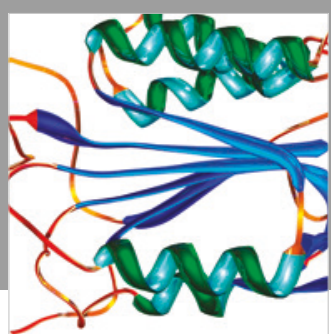

Disease Markers
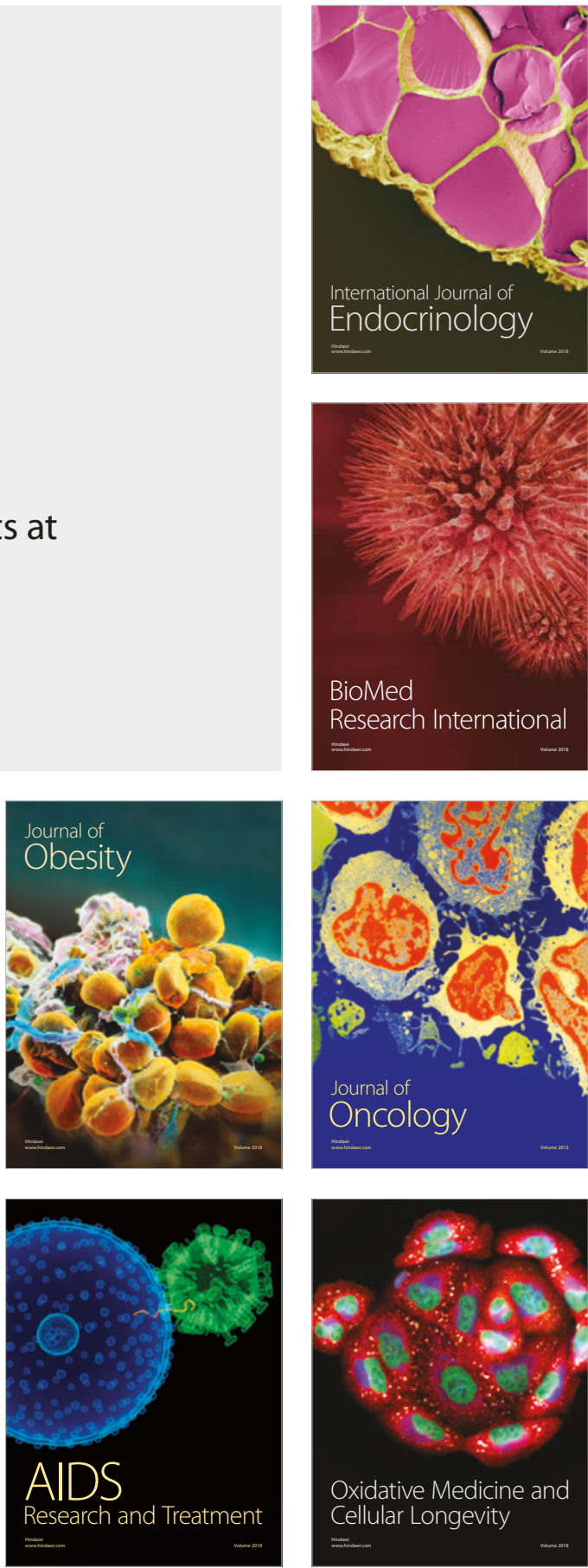